\title{
Size Control and Stability Study of Zeolitic Imidazolate Framework-8 to Prepare Mixed Matrix Membrane
}

\author{
Peng Chee Tan, ${ }^{*}$ Boon Seng Ooi, Abdul Latif Ahmad and Siew Chun Low \\ School of Chemical Engineering, Universiti Sains Malaysia, Engineering Campus, \\ 14300 Nibong Tebal, Pulau Pinang, Malaysia \\ *Corresponding author: peng_chee91@hotmail.com
}

Published online: 15 February 2017

To cite this article: Tan, P. C. et al. (2017) Size control and stability study of zeolitic imidazolate framework-8 to prepare mixed matrix membrane. J. Phys. Sci., 28(Supp. 1), 215-226, https://doi.org/10.21315/jps2017.28.s1.14

To link to this article: https://doi.org/10.21315/jps2017.28.s1.14

\begin{abstract}
Zeolitic imidazolate framework-8 (ZIF-8) with large particle size possesses significant gas transport resistance, which minimises its efficiency in improving the membrane gas separation performance. Hence, size control of ZIF-8 is of great research interest. In this work, different 2-methylimidazole (Hmim) concentration (molar ratio $\mathrm{Zn}$ : Hmim $=1: 8$ or 1:70) and synthesis solvent (methanol or deionised water) were used to regulate the ZIF-8 particle size. Smaller ZIF-8 particles were produced when the reaction was carried out at high Hmim concentration with short reaction time ( $\leq 2 \mathrm{~min})$ or when methanol was used as the synthesis solvent. It is postulated that the ZIF-8 particles are more likely to exist in the form of aggregates in the membrane rather than individual particles based on the dynamic light scattering (DLS), scanning electron microscopy (SEM) and transmission electron microscopy (TEM) analysis. As particle agglomeration was contributed by both particle drying and instability of ZIF-8 in THF, it was recommended to store the ZIF-8 in methanol suspension prior to usage.
\end{abstract}

Keywords: Colloidal stability, ZIF-8, imidazole linker, solvent effect, agglomeration

\section{INTRODUCTION}

In recent years, polymeric membrane has experienced extensive expansion for the application in gas separation due to its excellent processability. ${ }^{1-3}$ However, the separation performance of the polymeric membranes has reached a performance bottleneck because it seldom surpasses the trade-off between permeability and selectivity, as indicated by the Robeson plot. ${ }^{4}$ Recently, mixed matrix membrane 
(MMM), which comprises of inorganic particles dispersed within the continuous polymer matrix, has emerged as a promising route to enhance the gas transport properties through the membrane. By principle, MMM combines the outstanding processability of the polymer and the superior gas separation performance of the inorganic particles. 5,6

In the field of gas separation using MMM, zeolitic imidazolate framework-8 (ZIF8 ) has attracted considerable attention. ZIF-8 possesses large cages with the size of $11.6 \AA$ that are accessible through the small apertures that have the diameters of $3.4 \AA .^{7}$ Its pore size, which is in close proximity to the kinetic diameter of common gases, makes it highly advantageous for gas separation especially in the separation of $\mathrm{CO}_{2}(3.3 \AA)$ from other gases such as $\mathrm{N}_{2}(3.64 \AA)$ and $\mathrm{CH}_{4}(3.8 \AA)^{8}$ due to the notable molecular sieving effect. Besides, the large pore volume and high $\mathrm{CO}_{2}$ adsorption capacity ${ }^{9}$ demonstrated by the ZIF-8 also help in enhancing the gas separation efficiency. For example, Dai et al. showed that 17 vol\% of ZIF$8 /$ Ultem ${ }^{\circledR}$ hybrid hollow fibre membrane had improved both the $\mathrm{CO}_{2}$ permeability and $\mathrm{CO}_{2} / \mathrm{N}_{2}$ ideal selectivity by $85 \%$ and $20 \%$ respectively. ${ }^{5}$

It is well known that a ZIF-8 with large particle size increases the diffusion resistance, which directly restricts the gas transport through the membrane. ${ }^{10}$ Hence, the size control of ZIF-8 is necessary for its effective application in gas separation. The particle size of ZIF-8 can be regulated by different strategies including incorporation of additives, ${ }^{10,11}$ alteration of reactant ratio ${ }^{12}$ and adjustment of reaction time. ${ }^{13}$ In the work carried out by Tanaka et al., the ZIF-8 size in the range of $0.32-3.4 \mu \mathrm{m}$ was achieved by adjusting the imidazole/Zn molar ratio from 40 to 100 with the reaction occurring in an aqueous system. ${ }^{12}$ In their study, higher monodispersity was acquired at higher imidazole/Zn molar ratio. ${ }^{12}$ Furthermore, Venna et al. demonstrated a simple way of ZIF-8 size control by manipulating the reaction time. ${ }^{13}$ With the reaction time of $10 \mathrm{~min}$ to $24 \mathrm{~h}, \mathrm{ZIF}-8$ in the size of $50-500 \mathrm{~nm}$ were obtained. ${ }^{13}$

To the authors' best knowledge, there is lack of literature report on the role of synthesis solvent in controlling the ZIF-8 particle size. In this work, ZIF-8 was synthesised using methanol or water under similar reaction protocol. In most reports, high imidazole concentration $(\mathrm{Zn}: \mathrm{Hmim}$ molar ratio $=1: 70)$ was employed in the aqueous system to produce ZIF-8. Hence, the effect of low imidazole concentration ( $\mathrm{Zn}: \mathrm{Hmim}$ molar ratio $=1: 8)$ in tailoring the ZIF-8 particle size was also investigated. In addition, the colloidal stability of ZIF- 8 was examined as this will influence its application and effectiveness in the preparation of gas separation membrane. 


\section{EXPERIMENTAL}

\subsection{Materials}

Zinc nitrate hexahydrate $(\geq 99 \%)$ and 2-methylimidazole linker (Hmim, 99\%) were purchased from Sigma Aldrich (USA). The commercial ZIF-8 (Basolite ${ }^{\circledR}$ Z1200, BASF) was also obtained from Sigma Aldrich (USA) to compare with the synthesized ZIF-8. Methanol and tetrahydrofuran were attained from Merck (USA) and Fisher Scientific (UK) respectively. All chemicals were used as received without further purification.

\subsection{Synthesis of ZIF-8 Particles}

Solutions of $0.1 \mathrm{M}$ zinc nitrate hexahydrate and $0.8 \mathrm{M}$ 2-methylimidazole (Hmim) were prepared separately in equal volume of solvent (methanol or deionised water). Then, the zinc nitrate hexahydrate solution was added rapidly into the 2-methylimidazole solution. The final molar composition of the synthesis solution was $\mathrm{Zn}: H \operatorname{mim}=1: 8$. The reaction was carried out under agitation speed of 250 $\mathrm{rpm}$ at room temperature with reaction time ranging from $1 \mathrm{~min}$ to $4 \mathrm{~min}$. The particles formed were recovered by centrifugation at $3000 \mathrm{rpm}$ for $1 \mathrm{~h}$. Once separated, the particles were washed with fresh solvent twice via repeating cycle of sonication $(20 \mathrm{~min})$ and centrifugation $(3000 \mathrm{rpm}, 30 \mathrm{~min})$. A portion of the particles were dispersed in methanol to observe the particle size changes over time. The remaining particles were dried in vacuum oven at $65^{\circ} \mathrm{C}$ for $24 \mathrm{~h}$. As for the ZIF-8 synthesised in deionised water, the reaction occurred at a higher molar ratio of $\mathrm{Zn:Hmim}=1: 70$ was also performed.

\subsection{Size Stability Test of ZIF-8 Particles}

The particle size of ZIF-8 stored in methanol suspension was measured over the storage time. In order to study the size stability of ZIF-8 in THF, the ZIF-8 stored in methanol suspension was separated by centrifugation ( $3000 \mathrm{rpm}, 1 \mathrm{~h}$ ) and redispersed in $10 \mathrm{~mL}$ THF. The ZIF-8 in THF suspension then underwent sonication $\left(30 \mathrm{~min}, 50^{\circ} \mathrm{C}\right)$-stirring $(30 \mathrm{~min})$-sonication $\left(30 \mathrm{~min}, 50^{\circ} \mathrm{C}\right)$ cycle to obtain a uniformly dispersed suspension. Meanwhile, for the ZIF-8 stored in dry form, approximately $0.03 \mathrm{~g}$ of dry ZIF- 8 was suspended in $10 \mathrm{~mL}$ THF, in which the similar sonication-stirring-sonication cycle was applied before the size measurement. 


\subsection{Characterisations of ZIF-8 Particles}

The particle size of ZIF-8 was monitored using dynamic light scattering (DLS, Malvern Instruments Nanosizer, UK) in backscattering detection mode, in which the angle between the laser and the detector was $173^{\circ}$. A 6 ppm sample was used for the measurement to avoid multiple light scattering.

Scanning electron microscopy (SEM, Hitachi TM 3000, Japan) was used to observe the morphology of the ZIF-8 particles. The samples were coated with a thin gold conducting layer to obtain a clearer image.

The ZIF-8 structure was further observed using transmission electron microscopy (TEM, Philips CM12, Holland). The sample was prepared by evaporating few drops of ZIF-8 suspension onto the copper grids before the analysis.

\section{RESULTS AND DISCUSSION}

\subsection{Size Control of ZIF-8 Particles}

The role of 2-methylimidazole linker (Hmim) in controlling the size of ZIF8 particles was studied by adjusting the $\mathrm{Zn}: \mathrm{Hmim}$ molar ratio to $1: 8$ and $1: 70$, respectively. As shown in Figure 1, ZIF-8 synthesis at low molar ratio of 1:8 in aqueous system yielded a bigger size of ZIF- 8 particle than the higher molar ratio of $\mathrm{Zn}: \mathrm{Hmim}$ at 1:70 when the reaction was occurred in a rapid time (within $2 \mathrm{~min}$ ). However, when the reaction was prolonged beyond $2 \mathrm{~min}$, the trend was reversed.

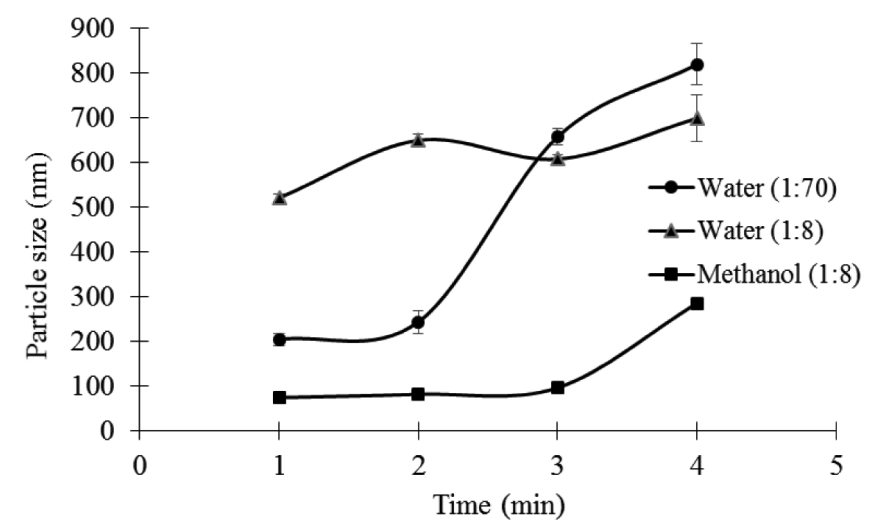

Figure 1: Particle size of ZIF-8 synthesised in water and methanol at different Zn:Hmim molar ratio as a function of reaction time. 
Fundamentally, the structural evolution of ZIF-8 starts with the nucleation stage. ${ }^{13} \mathrm{Hmim}$ deprotonates to provide imidazolate nitrogen atom, which is then coordinated with the $\mathrm{Zn}^{2+}$ ion to construct the neutral framework structure of ZIF8, as displayed in Figure 2. ${ }^{12}$ At the early stage of the reaction, the deprotonated imidazole was consumed for the nuclei formation rather than the particle growth. With higher Hmim concentration (molar ratio $=1: 70$ ), the deprotonation rate of imidazole was anticipated to increase. ${ }^{12}$ Henceforth, more nuclei were formed at the beginning of the reaction $(\leq 2 \mathrm{~min})$ at higher Hmim concentration. In this case, the overall ZIF-8 particle sizes were found smaller prior to the extensive growth of the particles. In contrast, less nuclei were formed when lower Hmim concentration (molar ratio $=1: 8$ ) was employed. Thus, the remaining deprotonated imidazole were able to attach to the nuclei produced, resulting in a larger ZIF-8 particle. However, the particle size of ZIF-8 synthesised at higher Hmim concentration (molar ratio $=1: 70$ ) was eventually bigger than that of lower Hmim concentration (molar ratio $=1: 8)$ at prolonged reaction time $(\geq 2 \mathrm{~min})$ as more deprotonated Hmim were available for the particle growth.

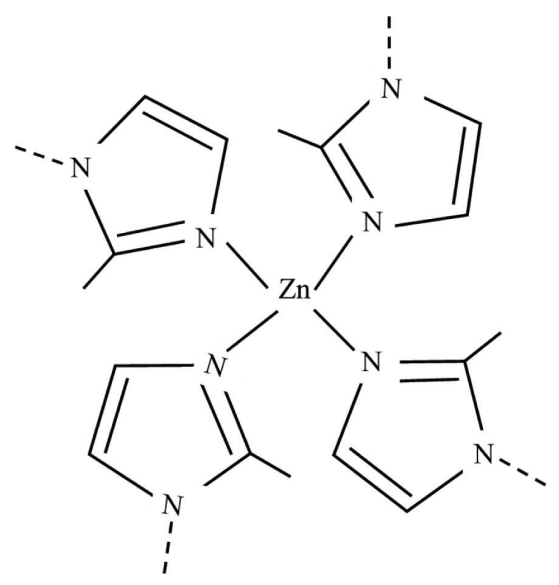

Figure 2: ZIF-8 framework structure.

In addition to Hmim concentration, the effect of solvent in regulating the ZIF-8 particle size could not be neglected as some of the solvents will favour the growth of ZIF-8. ${ }^{14}$ In this work, water and methanol were used as the solvents for ZIF-8 synthesis to compare the particles formed at constant $\mathrm{Zn}: H$ mim molar ratio of 1:8. During the synthesis, the reaction mixture prepared in water turns out cloudy faster than the reaction mixture prepared in methanol (data not shown). Hence, faster nuclei formation and particle growth was anticipated in the aqueous solution, which produced bigger size of ZIF-8 particle. This postulation was proven from the DLS results. As highlighted in Figure 1, the ZIF-8 produced from the water 
$(521 \mathrm{~nm})$ was 7 times bigger than that produced by using methanol as the solvent $(74 \mathrm{~nm})$ at 1 min reaction time. The result suggested that the growth of ZIF-8 particle was highly promoted by the water, attributed to its stronger ability of hydrogen bond donation $(\alpha=1.17) .{ }^{15}$ In contrast, the $\alpha$ of methanol was found to be 0.98 , which was lower than the $\alpha$ of water. ${ }^{15}$ The greater capability of water in generating hydrogen bonds has induced higher lability of the pyrimidinic proton, ${ }^{14}$ which facilitated the Hmim deprotonation process. Therefore, faster ZIF-8 growth was anticipated in the water, which produced bigger ZIF-8 particles.

Although three different synthesis conditions were adopted, it was not surprising that a similar trend in particle size evolutions were observed with increasing reaction time, as displayed in Figure 1. The size of ZIF-8 particles was increased gradually, followed by a drastic increment as the reaction progressed. As mentioned previously, this observation confirmed the initiated nucleation stage during the ZIF8 synthesis, which rationalised the slow increment in the particle size. Meanwhile, the significant increase of the ZIF-8 particle size in the latter stage of reaction was undoubtedly contributed by the particle growth. Among the three synthesis conditions, the reaction carried out in methanol at $\mathrm{Zn}$ : Hmim molar ratio of 1:8 was preferable as it produced smaller ZIF-8 particle for MMM fabrication, with the size ranging from $74-285 \mathrm{~nm}$.

\subsection{Size Stability of ZIF-8 Particles}

As for the membrane gas separation, the large sizes of ZIF-8 particles may induce significant gas transport resistance through the membrane. ${ }^{10}$ In view of the particle size requirement, ZIF-8 produced at 3 min reaction time $(96 \mathrm{~nm})$ in methanol solvent was chosen to be incorporated into the MMM. In this context, the size stability of the ZIF-8 over storage time is a critical issue to be addressed as the ZIF-8 particles might agglomerate during the storage period.

Table 1 indicates the size stability of ZIF- 8 stored in two different approaches. The first storage method is the direct dispersion of the recovered ZIF-8 in methanol solvent. It was interesting to note that the ZIF-8 stored in methanol suspension (without drying, 215-234 nm) was found slightly larger than the as-synthesised ZIF-8 $(96 \mathrm{~nm})$. This is reflected with a slow particle growth still occurring during the solid recovery process. In overall, the ZIF- 8 particles were stable over the storage period, with the particle sizes ranging from $215-234 \mathrm{~nm}$. However, it is of interest to determine the particle size of ZIF-8 in the casting solvent (tetrahydrofuran, THF) as it more closely represents the dispersion level of ZIF-8 particles in the membrane. Hence, the ZIF-8 in the stable methanol suspension was separated and dispersed in THF. Unfortunately, DLS result demonstrated ZIF-8 hydrodynamic 
size of $5276 \mathrm{~nm}$ when dispersed in THF. This revealed the instability of ZIF-8 in THF, where there is high possibility of particle agglomeration during the membrane synthesis. The second storage method is to keep the ZIF-8 in dry form. Over the storage time, the ZIF-8 showed a particle size of $<3000 \mathrm{~nm}$ when it was dispersed in THF (Table 1). In comparison to the as-synthesised ZIF-8 particle size of 96 $\mathrm{nm}$, the significantly larger ZIF-8 particles during the storage period indicated the possibility particle agglomeration.

Table 1: The particle size of ZIF-8 stored in different ways.

\begin{tabular}{ccc}
\hline \multirow{2}{*}{ Time } & \multicolumn{2}{c}{ Particle size $(\mathrm{nm})$} \\
\cline { 2 - 3 } & Direct dispersion of ZIF-8 in methanol & Dispersion of dry ZIF-8 in THF* \\
\hline Day 1 & 556 & 2473 \\
Day 2 & 222 & 2136 \\
Day 3 & 215 & 1693 \\
Day 4 & 234 & 2741 \\
\hline
\end{tabular}

*ZIF-8 was stored in dry form. The dispersion of dry ZIF-8 in THF was prepared each day before the size measurement.

Particle agglomeration is a major concern in the advancement of MMM. However, DLS is a physical technique that can only provide the particle size based on light scattering. The particle size measurement obtained cannot clearly indicate whether the sample is in the form of individual particles or aggregates. Therefore, the ZIF8 particles were further examined using SEM to have a better insight on their physical appearance. As demonstrated in Figure 3(a), lumps of ZIF-8 particles with irregular size were noted when the dry ZIF- 8 were dispersed in THF. The co-existence of both large and small clusters is expected to create an uneven interfacial stress between the ZIF-8 and the polymer matrix, which will weaken the mechanical strength of the resulting MMM.

Meanwhile, the SEM image also revealed the aggregated form of the commercial ZIF-8 (Basolite ${ }^{\circledR}$ Z1200), as denoted in Figure 3(b). Although the DLS measurements showed that the particle size of the commercial ZIF-8 $(2338 \mathrm{~nm})$ in THF was comparable to the self-synthesised ZIF-8 stored in dry form (2136$2741 \mathrm{~nm}$ ), the aggregated size of the commercial ZIF-8 was more homogeneous. This reflected that the commercial ZIF- 8 stands a higher chance to produce MMM without interfacial defects. Regardless of the commercial or self-synthesised ZIF8 , the SEM images showed that the ZIF-8 is most likely to distribute in the form of aggregates rather than individual particles in the membrane (Figure 4). Henceforth, further works are required to investigate the effectiveness of ZIF-8 aggregates in the MMM for gas separation application. 


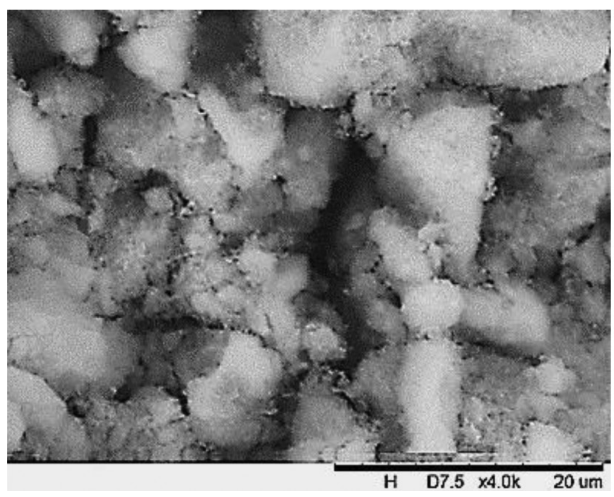

(a)

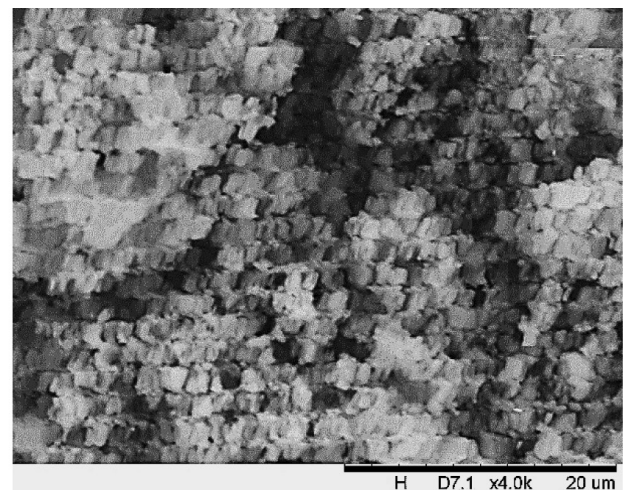

(b)

Figure 3: SEM images of (a) dry synthesised ZIF-8 particles dispersed in THF, and (b) commercial Basolite ${ }^{\circ}$ Z1200 ZIF-8.

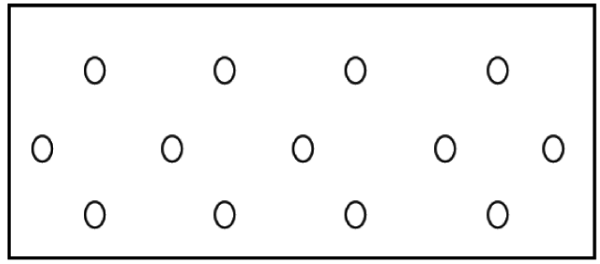

Distribute in the form of individual particle

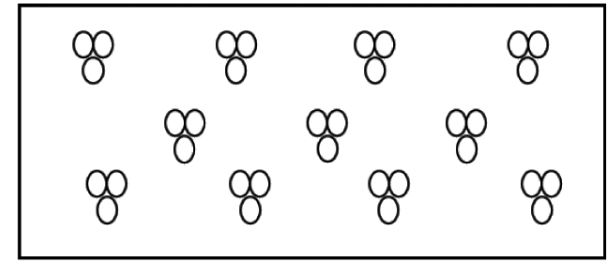

Distribute in the form of aggregates

Figure 4: Distribution of ZIF-8 particles in the membrane.

Despite the fact that the commercial ZIF-8 might be more preferable for MMM synthesis, efforts to improve the self-synthesised ZIF-8, especially in tackling the particle agglomeration, could not be neglected due to the easy tuning of particle size and morphology for particular application. As aforementioned, it was expected that the ZIF-8 was unstable in THF as the ZIF-8 particle size increased dramatically when it was redispersed in THF (increased from 215-234 nm in methanol suspension to $5276 \mathrm{~nm}$ in THF). However, based on the SEM result in Figure 3(a), in which the aggregated form was obtained when the dry ZIF-8 particles were dispersed in THF, ZIF-8 agglomeration was possibly contributed by both the instability of ZIF-8 in THF and the particle drying. Hence, the SEM images of ZIF-8 stored in two different conditions: (1) in methanol suspension and (2) recovered from the methanol suspension and redispersed in THF, were examined to investigate the root cause of ZIF-8 agglomeration. Surprisingly, undistinguishably large ZIF-8 clusters (Figure 5(a) and (b)) were observed even though the ZIF-8 in Figure 5(a) 
was not being dispersed in THF. As the SEM analysis requires dry samples, the results highlighted the significant role of drying in causing the ZIF-8 aggregation (the ZIF-8 was kept in suspension form before the analysis). It was postulated that drying promoted the formation of strong covalent bonding between different ZIF-8 particles, which resulted in particle agglomeration. ${ }^{16}$

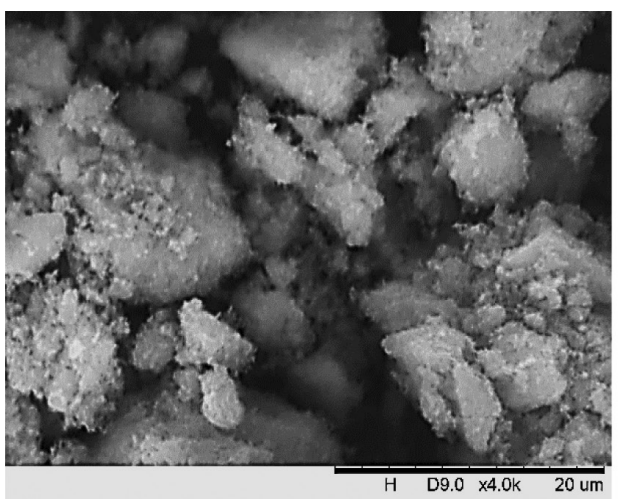

(a)

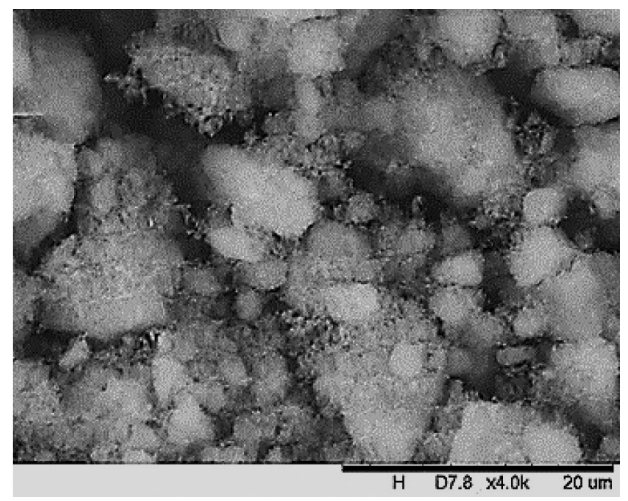

(b)

Figure 5: SEM images of the synthesised ZIF-8 particles (a) dispersed in methanol suspension and (b) redispersed in THF from the methanol suspension.

The drying effect on particle agglomeration was further verified by the TEM analysis. As shown in Figure 6(a), the ZIF-8 in methanol suspension (not subjected to drying) demonstrated regular particle size and was well dispersed, in which the particle agglomeration was absent. In comparison to its aggregated form denoted by the SEM image (Figure 5(a)), the result stressed that it was drying that caused the serious particle agglomeration. In the meantime, the stability of ZIF-8 in THF (casting solvent) is also of great concern. It was found that a portion of the synthesised ZIF-8 agglomerated when dispersed in THF (Figure 6(b)). This signified that the ZIF-8 was incompatible with the THF, in which the ZIF-8 might exist as aggregated form in the membrane. However, some individual ZIF-8 particles could still be observed from the TEM image of the self-synthesised ZIF-8 in THF (Figure 6(b)). In contrast, a more severe ZIF-8 agglomeration was noted when the commercial Basolite ${ }^{\circledR}$ Z1200 ZIF-8 was dispersed in THF (Figure 6(c)). Since the commercial ZIF-8 was initially in dry form, the result again emphasised that the severe agglomeration was attributed to the combined effects of both particle drying and instability of ZIF-8 in THF. In overall, it is recommended to store the ZIF-8 in methanol suspension instead of dry form prior to its usage. Besides, more efforts should be focused on the ZIF-8 dispersion in the casting solvent to ensure its successful integration into the mixed matrix membrane. 


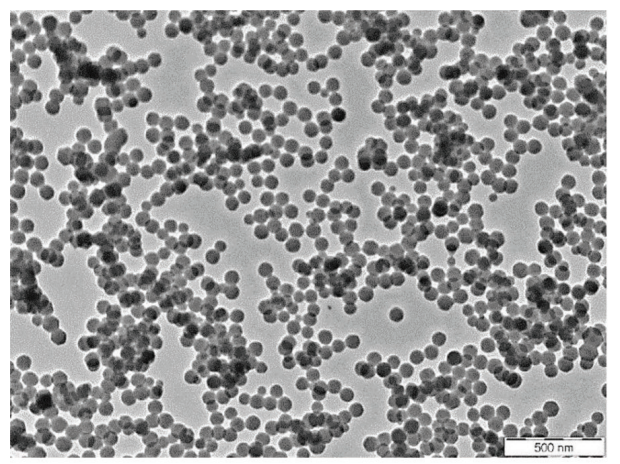

(a)

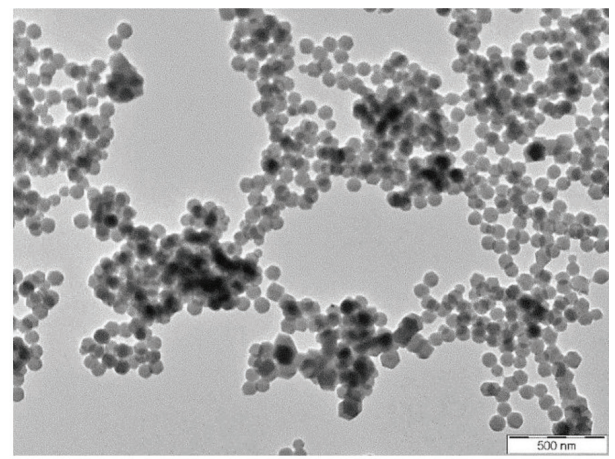

(b)

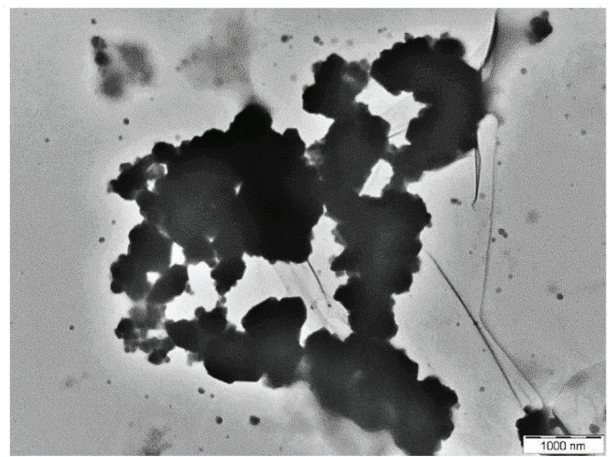

(c)

Figure 6: TEM images of the synthesised ZIF-8 particles (a) dispersed in methanol suspension; (b) redispersed in THF from the methanol suspension; (c) TEM image of the commercial Basolite ${ }^{\circledR}$ Z1200 ZIF-8 dispersed in THF.

\section{CONCLUSION}

The ZIF-8 particle size was successfully tailored by manipulating the 2-methylimidazole (Hmim) concentration and the synthesis solvent (methanol or deionised water). Within short reaction time ( $\leq 2 \mathrm{~min})$, smaller ZIF-8 particles were produced at higher Hmim concentration (molar ratio $\mathrm{Zn}: \mathrm{Hmim}=1: 70$ ) in the aqueous reaction system attributed to the increased nucleation rate. Besides, the formation of smaller ZIF-8 particles, in the range of 74-285 nm, was realised by employing methanol as the synthesis solvent due to its weaker ability of hydrogen bond donation during the reaction. SEM images showed the agglomeration of ZIF-8 particles, which implied the high possibility of ZIF-8 to be distributed in the form of aggregates rather than individual particles in the membrane. It was found that the ZIF-8 agglomeration was mainly caused by the particle drying and 
the instability of ZIF-8 in THF. Hence, it is preferable for ZIF-8 to be stored in methanol suspension rather than dry form to minimise the agglomeration problem. For successful integration of ZIF-8 in mixed matrix membrane, further work on improving the compatibility between ZIF-8 and casting solvent (THF) is needed.

\section{ACKNOWLEDGEMENTS}

The authors appreciate the Long Term Research Grant Scheme (LRGS) (304/ PJKIMIA/6050296/U124) and Membrane Science and Technology Cluster of USM for the financial supports. Peng Chee Tan is financially sponsored by the Human Life Advancement Foundation (HLAF) Scholarships.

\section{REFERENCES}

1. Kraftschik, B. et al. (2013). Dense film polyimide membranes for aggressive sour gas feed separations. J. Membr. Sci., 428, 608-619, https://doi.org/ 10.1016/j.memsci.2012.10.025.

2. Li, Y., Cao, C., Chung, T.-S. \& Pramoda, K. P. (2004). Fabrication of duallayer polyethersulfone (PES) hollow fiber membranes with an ultrathin dense-selective layer for gas separation. J. Membr. Sci., 245(1-2), 53-60, https://doi.org/10.1016/j.memsci.2004.08.002.

3. Ahmad, A. L. et al. (2014). A cellulose acetate/multi-walled carbon nanotube mixed matrix membrane for $\mathrm{CO}_{2} / \mathrm{N}_{2}$ separation. J. Membr. Sci., 451, 55-66, https://doi.org/10.1016/j.memsci.2013.09.043.

4. Robeson, L. M. (2008). The upper bound revisited. J. Membr. Sci., 320(1-2), 390-400, https://doi.org/10.1016/j.memsci.2008.04.030.

5. Dai, Y. et al. (2012). Ultem $\AA / Z I F-8$ mixed matrix hollow fiber membranes for $\mathrm{CO}_{2} / \mathrm{N}_{2}$ separations. J. Membr. Sci., 401-402, 76-82, https://doi.org/10. 1016/j.memsci.2012.01.044.

6. Ordonez, M. J. C. et al. (2010). Molecular sieving realized with ZIF-8/ Matrimid ${ }^{\circledR}$ mixed-matrix membranes. J. Membr. Sci., 361(1-2), 28-37, https://doi.org/10.1016/j.memsci.2010.06.017.

7. Kida, K. et al. (2013). Formation of high crystalline ZIF-8 in an aqueous solution. CrystEngComm., 15(9), 1794-1801, https://doi.org/10.1039/ c2ce26847g.

8. Li, K. et al. (2008). Multifunctional microporous MOFs exhibiting gas/ hydrocarbon adsorption selectivity, separation capability and threedimensional magnetic ordering. Adv. Funct. Mater., 18(15), 2205-2214, https://doi.org/10.1002/adfm.200800058. 
9. Venna, S. R. \& Carreon, M. A. (2009). Highly permeable zeolite imidazolate framework-8 membranes for $\mathrm{CO}_{2} / \mathrm{CH}_{4}$ separation. J. Am. Chem. Soc., 132(1), 76-78, https://doi.org/10.1021/ja909263x.

10. Pan, Y. et al. (2011). Tuning the crystal morphology and size of zeolitic imidazolate framework-8 in aqueous solution by surfactants. CrystEngComm., 13(23), 6937-6940, https://doi.org/10.1039/c1ce05780d.

11. Gross, A. F., Sherman, E. \& Vajo, J. J. (2012). Aqueous room temperature synthesis of cobalt and zinc sodalite zeolitic imidizolate frameworks. Dalton Trans., 41(18), 5458-5460, https://doi.org/10.1039/c2dt30174a.

12. Tanaka, S. et al. (2012). Size-controlled synthesis of zeolitic imidazolate framework-8 (ZIF-8) crystals in an aqueous system at room temperature. Chem. Lett., 41(10), 1337-1339, https://doi.org/10.1246/cl.2012.1337.

13. Venna, S. R., Jasinski, J. B. \& Carreon, M. A. (2010). Structural evolution of zeolitic imidazolate framework-8. J. Am. Chem. Soc., 132(51), 1803018033, https://doi.org/10.1021/ja109268m.

14. Bustamante, E. L., Fernández, J. L. \& Zamaro, J. M. (2014). Influence of the solvent in the synthesis of zeolitic imidazolate framework-8 (ZIF-8) nanocrystals at room temperature. J. Colloid Interf. Sci., 424, 37-43, https:// doi.org/10.1016/j.jcis.2014.03.014.

15. Marcus, Y. (1993). The properties of organic liquids that are relevant to their use as solvating solvents. Chem. Soc. Rev., 22(6), 409-416, https://doi.org/10.1039/cs9932200409.

16. Cravillon, J. et al. (2009). Rapid room-temperature synthesis and characterization of nanocrystals of a prototypical zeolitic imidazolate framework. Chem. Mater., 21(8), 1410-1412, https://doi.org/10.1021/ cm900166h. 